Journal of Engineering Sciences, Assiut University, Vol. 36, No. 1, pp. 115-130, January 2008

\title{
PROBABILITY CHARACTERISTICS OF CONCENTRATION FLUCTUATIONS IN PLUME DISPERSAL
}

\author{
Mohamed F. Yassin \\ Faculty of Engineering, Assiut University, Assiut-71516, Egypt \\ E-mail: mfy64@yahoo.com/mfy@aun.edu.eg
}

(Received November 5, 2007 Accepted December 9, 2007)

The probability characteristics of the concentration fluctuation in pollutant plume dispersion in a neutral stratified atmospheric boundary layer were investigated experimentally in a wind tunnel experiment. It was examined using a tracer gas without buoyancy, which emitted from a fixed point in the ground surface. The diffusion fields in the boundary layer were examined using an isolated building model. Instantaneous concentration fluctuations were measured along the mean plume centerline at various downwind distances from the source at different heights with the frequency of $1 \mathrm{KHz}$. Probabililistic analysis was performed on the concentration fluctuation, and results were presented for probability density functions (PDF) of mean concentration, fluctuation intensity and crosswind mean-plume dispersion. Furthermore, the effects of turbulence intensity on the statistical nature of concentration fluctuations were presented and discussed. Probability density functions of the concentration fluctuation data have shown a significant nonGaussian behavior. The lognormal distribution appeared to be the best fit to the shape of actual probability density functions of fluctuating concentration measured in the boundary layer wind flows in a wind tunnel. The plume dispersion PDF near the source was observed shorter than that those far from the source.

KEYWORDS: Atmospheric dispersion; Concentration fluctuation; Probability distribution; Wind tunnel experiment

\section{INTRODUCTION}

The boundary layer of the atmosphere is characterized by relatively high turbulence, with fluctuations in one-second averages of variables such as wind speed typically having magnitudes roughly equal to 10 to $100 \%$ of the mean value. When pollutants are emitted into this boundary-layer velocity field, they are carried out by turbulent eddies and also are observed to exhibit fluctuations at the same order of magnitude as their mean value. Therefore, these fluctuations are of great practical importance when the pollutant is highly toxic or flammable material. Most dispersion models are capable of predicting the mean pollutant concentration, but provide no guidance on the probability of fluctuations from this mean (Hanna and Insley, 1989).

The study of concentration fluctuations in dispersing plumes of pollutants in urban environment has resulted considerable advance in understanding the detailed structure of plumes in recent years. An understanding of concentration fluctuation is 
important for a number of practical applications and out of these is the largest peak concentration, which is of greatest interest. Several experiments (Myle and Mason, 1991; Cheung and Melbourne, 2000; Ma et al., 2005) have described tracer experiments using fast response concentration detectors. Those studies describe the turbulent fluctuations of concentrations at fixed points downstream of continuously emitting source. The results have given statistical descriptions of the concentration fluctuations under relatively simple atmospheric conditions in terms of, the standard deviation of concentration and the concentration probability density function (PDF).

Study of probability distribution of fluctuating concentration has been hampered by a lack of suitable instrumentation. Some of the initial attempts at both predicting concentration fluctuations and measuring fluctuations in ground level plumes have again emphasized the need for thorough experimentation. Recently, several studies have been completed for the probability distribution of concentration fluctuations. Sykes (1984), Hanna (1984), Sawford et al. (1985), Sawford (1987) and Diner et al. (1988) have agreed that the distribution of concentration fluctuation is skewed towards higher values, but disagree on the exact form of optimum distribution function. Furthermore, they have suggested analytical expressions for the mean and the standard deviation of the concentration fluctuations and found that the fluctuation intensity varies with distance from the source, position within plume, average time, and the time scale of atmospheric turbulence. However, observations can not provide any information on eddy size that causes the observed fluctuations. Sykes (1988) discussed how knowledge of concentration fluctuations may be used in the evaluation of dispersion models to give an estimate of inherent variability and errors in predictions. In addition, a few probability distributions with field and wind tunnel experiments have been completed in which series of pollutant concentrations have been observed (Fackrell and Robins, 1982; Hanna and Insley, 1989). Yee and Biltoft (2004) have presented a detailed picture of the behavior of concentration fluctuation statistics such as concentration standard deviation ratio, probability density function, power spectra and so on the domination motions in the array plume

The probability density function of concentration in an atmospheric plume is an important quantity used to describe and discuss environmental diffusion. The plume concentration PDF forms the basis for the definition and computation of a number of relevant parameters intervening in various practical problems. It has many applications. Yee and Chan (1997) have assessed the level of harm from toxic materials that requires the frequency of concentration peaks that exceed the critical threshold. Weil et al., (2002) have been proposed a number of very different probability density function forms, and they have great efforts to measure, model and understand the concentration of the probability density function However, despite this enormous effort, there are still no general mechanisms of the PDF that provides the best fit to plume concentration data over a wide range of experimental conditions.

This paper reports the analysis of probability characteristics of concentration fluctuations in plume dispersion in the atmospheric boundary layer wind tunnel experiments under neutral conditions. With this in mind, the probability density function of the instantaneous plume concentration are measured and applied to analyze data at various downwind distances from the source for a number of different heights. The aim of this paper is to improve understanding of the mechanisms of concentration 
fluctuations in plume dispersion emitted from a continuously steady source at fixed point in an urban Environment.

\section{EXPERIMENTAL DETAILS}

The experiments considered in this paper were conducted on the Atmospheric Diffusion Simulation Wind Tunnel. It is of open circuit type. The effective working section is $1 \mathrm{~m}$ high, $1.2 \mathrm{~m}$ wide and $4 \mathrm{~m}$ long following a $10 \mathrm{~m}$ long development section just downstream of the boundary layer. A turbulent boundary layer for the neutral atmospheric conditions in an urban environment was simulated on a scale of 1:300. The obstacles used in the flow and diffusion fields was isolated building model with height $200 \mathrm{~mm}$, width $100 \mathrm{~mm}$ and length $100 \mathrm{~mm}$.

A simulated atmospheric boundary layer was obtained by using a combination of barrier wall, elliptic vortex generators and roughness elements on the floor of the tunnel as shown in the schematic diagram of Fig.1. This combination of barriers, vortex generators and roughness elements produced a simulated atmospheric boundary layer with a normal depth, $\delta$, of $0.6 \mathrm{~m}$ and a free stream wind speed, $U_{\infty}$ of $1.3 \mathrm{~ms}^{-1}$. The power law $U \propto Z^{n}$ was applied to the vertical wind profile. The typical value of $1 / 4$ for $n$ in an urban area was employed. For details of the simulated turbulent boundary layer in the wind tunnel, refer to Yassin et al. (2006).

The rotation speed of the wind tunnel fan was kept constant during the experiment to achieve a steady stream velocity of about $1.3 \mathrm{~ms}^{-1}$. This is monitored with a pitot-tube and a split-fiber probe, which was $1.25 \mathrm{~mm}$ long and $70 \mu \mathrm{m}$ in diameter. The anemometer signals were digitized at a sampling rate of $1 \mathrm{kHz}$. A $120 \mathrm{~s}$ average time was used for all mean measurements. Measurements of wind velocity and turbulence were made simultaneously using Hot Wire Anemometer. Turbulence measurements were used in the analysis for normalization of results and assessment of surface-layer stability, for more details refer to Yassin et al. (2006).

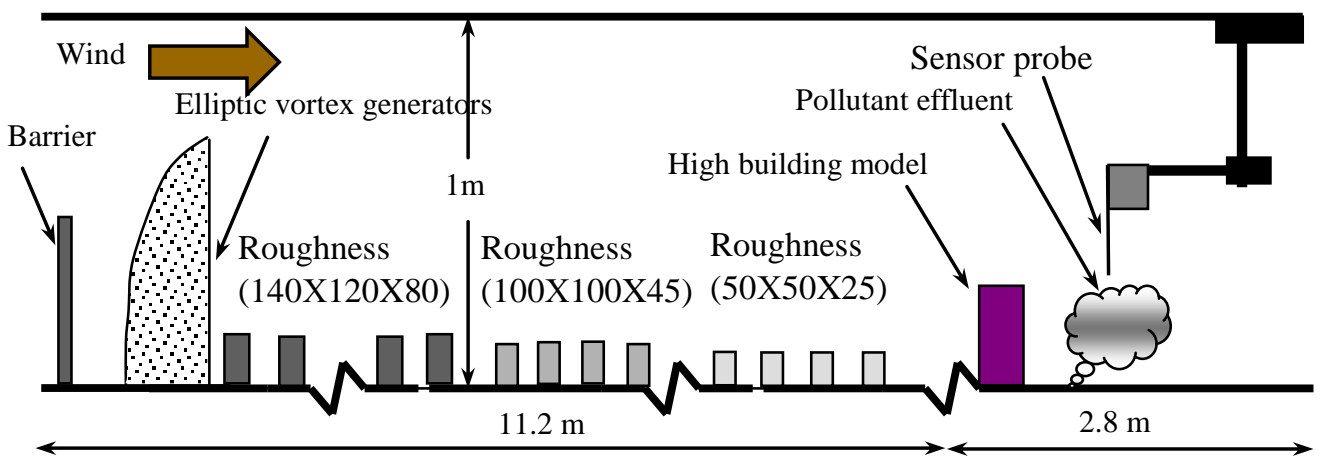

Fig. 1 Schematic diagram of the wind tunnel experiments

The concentration detector used was the High Response Total Hydrocarbon Analyzer (THC-1A). It is a Flame Ionization Detector (FID) based instrument giving high linearity and has a sampling system designed to provide fast response. The FID was calibrated at the beginning of each day by connecting it to a gas supply at atmospheric pressure which could be switched between two standard samples of tracer 
in zero grade air in concentration by 0 and $1000 \mathrm{ppm}$. The FID have a response time to a step change in concentration of $1 \mathrm{ppm}$ of about $0.05 \mathrm{~s}$ from 10 to $90 \%$ response, which is fast enough for most practical applications. In accordance with the purpose of the present investigation, a tracer gas was to be neutrally buoyant and released from the fixed point in the ground surface. The tracer gas chosen, in this case is ethylene, $\mathrm{C}_{2} \mathrm{H}_{4}$, which was emitted from the fixed point. The exhaust gas velocity is $0.27 \mathrm{U}_{\mathrm{H}}\left(\mathrm{U}_{\mathrm{H}}\right.$ is velocity at height building). Tracer was controlled using a constant-pressure regulator and a rotameter to ensure a constant flow-rate and was released through the pipeline attached to the carriage system in the wind tunnel. The samples were routed to the FID which produced output voltages linearly related to concentration. The output voltage from the FID was sampled by minicomputer system at a rate of $1 \mathrm{KHz}$ over averaging time of $120 \mathrm{~s}$, which yield reasonably stable values of mean concentration.

\section{DISCUSSION AND ANALYSIS OF RESULTS}

The log-normal distribution is widely used to represent long-term urban air quality statistics. It is successful in this instance since the extended area in the source, the main dilution mechanism is ventilation due to the mean wind speed which itself found to be log-normally distributed. The PDF of the lognormal distribution for concentration fluctuations is given by ( $\mathrm{Li}$ et al, 2005)

$$
f(c)=\frac{\exp \left(-0.5((\ln (|c|+\hat{c})-\mu) / \sigma)^{2}\right)}{\sqrt{2 \pi \sigma}(|c|+\hat{c})}
$$

Where the parameters, $\sigma$ and $\mu$ can be determined conveniently by calculating the mean and standard deviations of the natural logarithms of the data, and $\hat{c}$ is the positive crest factor.

The shape of a probability density function of the concentration fluctuation (c) can be characterized by its third and fourth central moments $\left(\mu_{3}\right.$ and $\left.\mu_{4}\right)$ relative to the standard deviation value $\left(\sigma_{\mathrm{c}}\right)$. The skewness coefficient, $S_{k}$, and the kurtosis coefficient, $K_{u}$, are defined, respectively, as

$$
\begin{aligned}
& S_{k}=\mu_{3} / \sigma_{c}^{3} \\
& K_{u}=\mu_{4} / \sigma_{c}^{4}
\end{aligned}
$$

Where

$$
\begin{gathered}
\sigma_{c}^{2}=\sum\left(c_{i}-\bar{c}\right)^{2} / n \\
\mu_{3}=\sum\left(c_{i}-\bar{c}\right)^{3} / n \\
\mu_{4}=\sum\left(c_{i}-\bar{c}\right)^{4} / n
\end{gathered}
$$

In which $c_{i}$ is instantaneous concentration fluctuation, $\bar{c}$ is the time mean concentration and $n$ is total number of samples.

For a normal or Gaussian distribution, skewness and kurtosis coefficient are equal to 0 and 3 , respectively. $S_{k}<0$ corresponds to skewness to the left, while $S_{\mathrm{k}}>0$ to the right. $K_{u}>3$ represents distributions more peaked than the Gaussian and $K_{u}<3$ characteristics distributions flatter than Gaussian.

The positive and negative crest factors are defined as 


$$
\begin{aligned}
& \hat{c}=\max (c-\bar{c}) / \sigma_{c} \\
& \check{c}=\min (c-\bar{c}) / \sigma_{c}
\end{aligned}
$$

It is usually more convenient to use the following reduced variety of fluctuation intensity $I$ when dealing with probability distribution, defined as

$$
I=\sigma_{c} / \bar{c}
$$

The crosswind mean-plume dispersion, $\sigma_{y}$ was determined by Gaussian plume width, calculated from the measured turbulence statistics using the formula (Mylne et al, 1992)

$$
\sigma_{y}=\sigma_{v} \frac{c f(x)}{U}
$$

where $f(x)$ is a correction factor whose is taken from Pasquill and smith (1983) and is close to 1.0 for $x \sim 100-300 \mathrm{~m}, \sigma_{v}$ is the standard deviation of the wind velocity in the lateral direction and $U$ is mean wind velocity in the longitudinal direction.
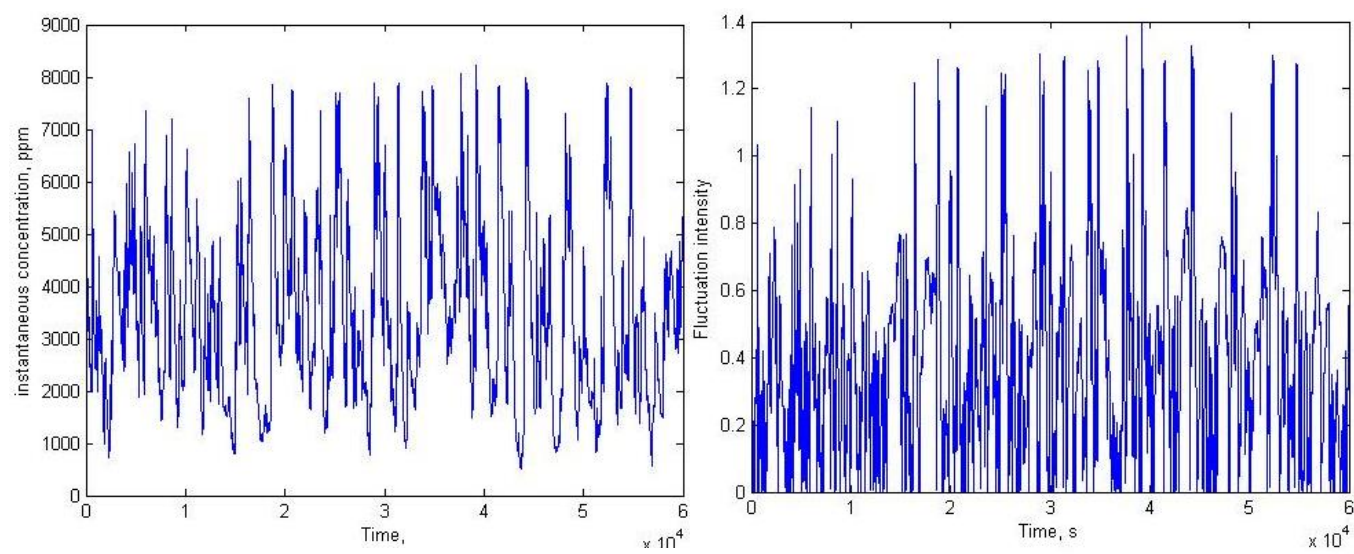

Fig. 2 The time series of instantaneous concentration and fluctuation intensity at $\mathrm{X} / \mathrm{H}=$ 0.375 for $\mathrm{Z} / \mathrm{H}=0.1$.

\subsection{Probability Distribution of Mean Concentration}

The basic concentrations was measured along the mean plume centerline in the wake region of the building model with various downwind distances $X / H$ from the source for different heights $Z / H$ through a vertical plume cross-section with the frequency of 0.5 $\mathrm{KHz}$ for 120 seconds. The non- dimensional downwind distances $X / H=0.375,0.625$ and 1.125 for different heights $Z / H=0.1,0.25,0.375$ and 0.75 were selected in order to illustrate the change in the PDF with downwind distance from the source and height above the ground. The flow obstacle model was located at $X / H=0$. The ground source was located at $X / H=0.125$ that has an inner diameter of $4 \mathrm{~mm}$. The plume is nonbuoyant with exit velocity less than the wind velocity. Fig. 2 illustrates an example of time series with the instantaneous concentration and fluctuation intensity, taken at $X / H$ $=0.375$ and $\mathrm{Z} / \mathrm{H}=0.1$. It was seen that the fluctuation intensity falls abruptly to a constant zero within $120 \mathrm{~s}$ of some largest peaks recorded, indicating that the edge of 
the meandering plume is quite well defined relative to the scale of the meandering. The time series of instantaneous concentration meandering motions can be clearly seen as simultaneously with onset of the peaks fluctuation intensity, the instantaneous concentration become more constant and the stronger fluctuation.

The probability distribution is an important character of the concentration fluctuations; this is because it might happen the exceeded level of toxic gasses might be found at a given location and point of time toxic level is exceeded, no matter what the mean value at that location is. Histograms of the instantaneous concentration fluctuation $c$ normalized by the mean concentration $\bar{c}$ were complied. Figs 3 to 5 display the measured PDF of $\mathrm{c} / \bar{c}$ along the mean plume centerline at various nondimensional downwind distances $X / H=0.375,0.625$ and 1.125 from the source for different heights $Z / H=0.1,0.25,0.375$ and 0.75 in the near wake region of the building model. The non-dimensional downwind distances for different heights were selected in order to illustrate the change in the shape of the PDF with downwind distance from the source and with height above the ground. Normal and lognormal probability densities are also plotted in these figures for comparison purpose. It is infer that, the lognormal distribution appeared to be the best fit to the shape of the actual probability density functions of concentration measured in the boundary layer wind flows in a wind tunnel. It is seen that the peak value of concentration PDF at the mean plume centerline were observed close to the source at the downwind of $X / H=$ 0.375 with heights $Z / H=0.1$ due to the higher turbulence mixing near the ground in the wake region. The longer tail of concentration PDF was observed at distance $X / H=$ 1.125 with height $Z / H=0.375$, while the shorter tail observed $X / H=0.375$ for $Z / H=$ 0.75. The tail of concentration PDF for heights far the ground surface was observed shorter than that those heights near the ground surface except at $X / H=1.125$. The wider shape of concentration PDF was observed for height $Z / H=0.75$ at distance $X / H$ $=0.375$. In the wake region of the building model, at height $Z / H=0.375$ at distance $X / H=0.375$, the peak concentration PDF was observed a wide sharp, because the value of concentration was high. In addition, the concentration PDF in narrow shape was observed far the source. A higher probability of observing concentrations close to $\mathrm{c} / \bar{c}=0.75$. As can be seen in these figures, the slope of concentration PDF near from the source is much steeper than that those near the source.

\subsection{Probability distribution of Fluctuation Intensity}

The parameter that frequently used to characterize the concentration fluctuation is fluctuation intensity. Figs. 6 and 8 display the probability density functions (PDF) of normalized fluctuation intensity $\sigma_{d} \bar{c}$ at various distances $X / H=0.375,0.625$ and 1.125 for different heights $\mathrm{Z} / \mathrm{H}=0.1,0.25,0.375$ and 0.75 , which was measured in the near wake region of the high rise building model. The lognormal distribution fits the actual PDF in the centre region much better than the normal. The fluctuation intensity PDF presented in these figures was clearly positive skewed. The fluctuation intensity PDF based on the experimental data are distinctly different from the normal distribution, particularly for both tails. The actual probability distributions have much longer positive tails. This indicates a much higher probability for the larger positive concentration than that predicted by a Gaussian PDF. However, the lognormal 
distribution represents a much better fit to the tails of the probability densities than the normal distribution. As the probability distribution in the region of the tail has a significant effect on the estimation of peak concentration fluctuations, it is important to give reasonably close representation of the actual probability distribution at the tail of the positive side. The experimental data exhibited a probability distribution more peaked at the centre than the Gaussian.

The evolution of skewness and kurtosis of the fluctuation intensity calculated based on the data obtained at $X / H=0.375,0.625$ and 1.125 for height $\mathrm{Z} / \mathrm{H}=0.1,0.375$ and 0.75 are shown in Table 1 . The skewness and kurtosis of the concentration fluctuation position provides information on the distribution of the plume as transported by the large-scale turbulent motion. As pointed out by Luhar et al (2000),
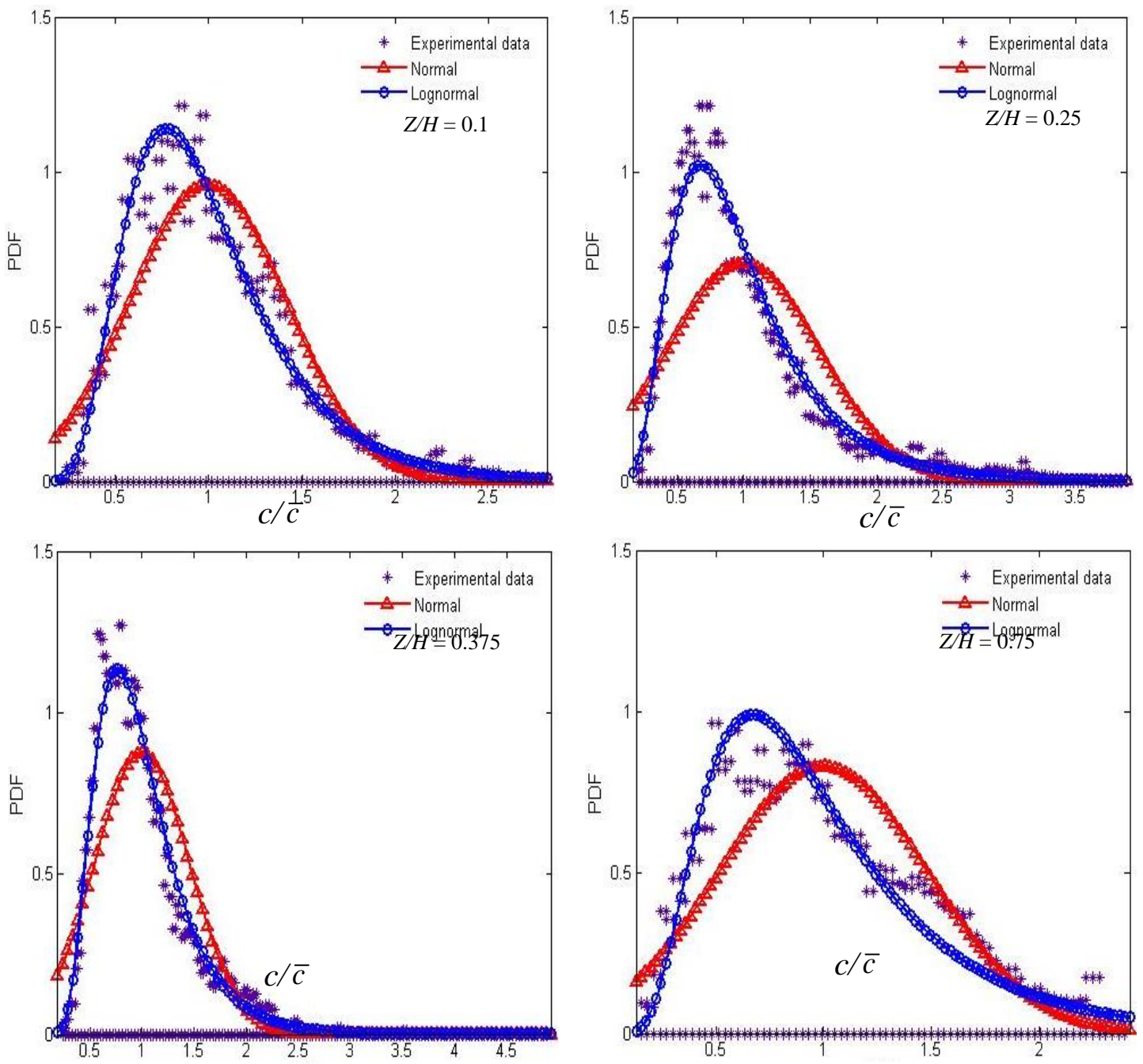

Fig. 3 Probability density for mean concentration measured at $\mathrm{X} / \mathrm{H}=0.375$ 

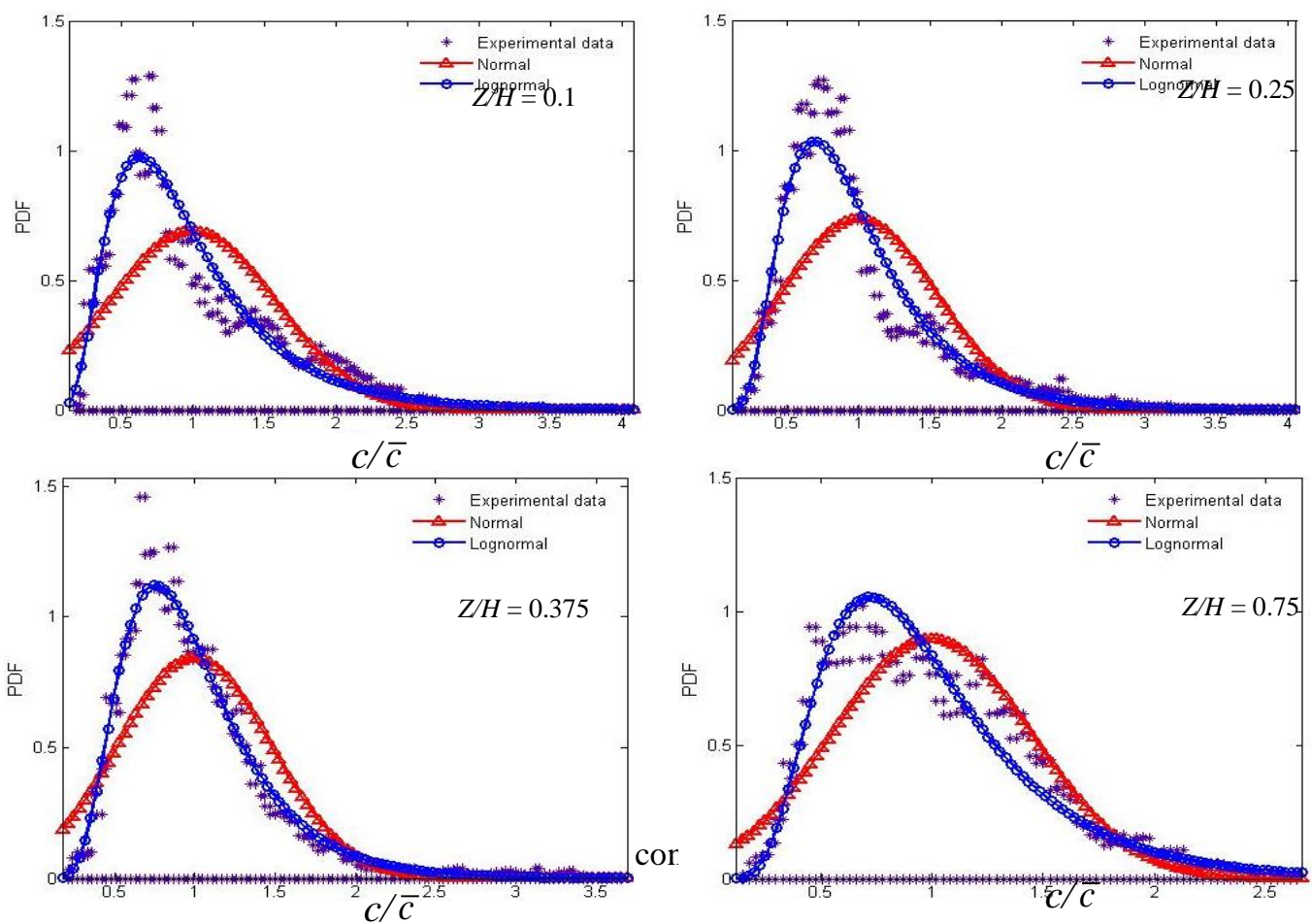

Fig. 4 Probability density for mean concentration measured at $\mathrm{X} / \mathrm{H}=0.625$
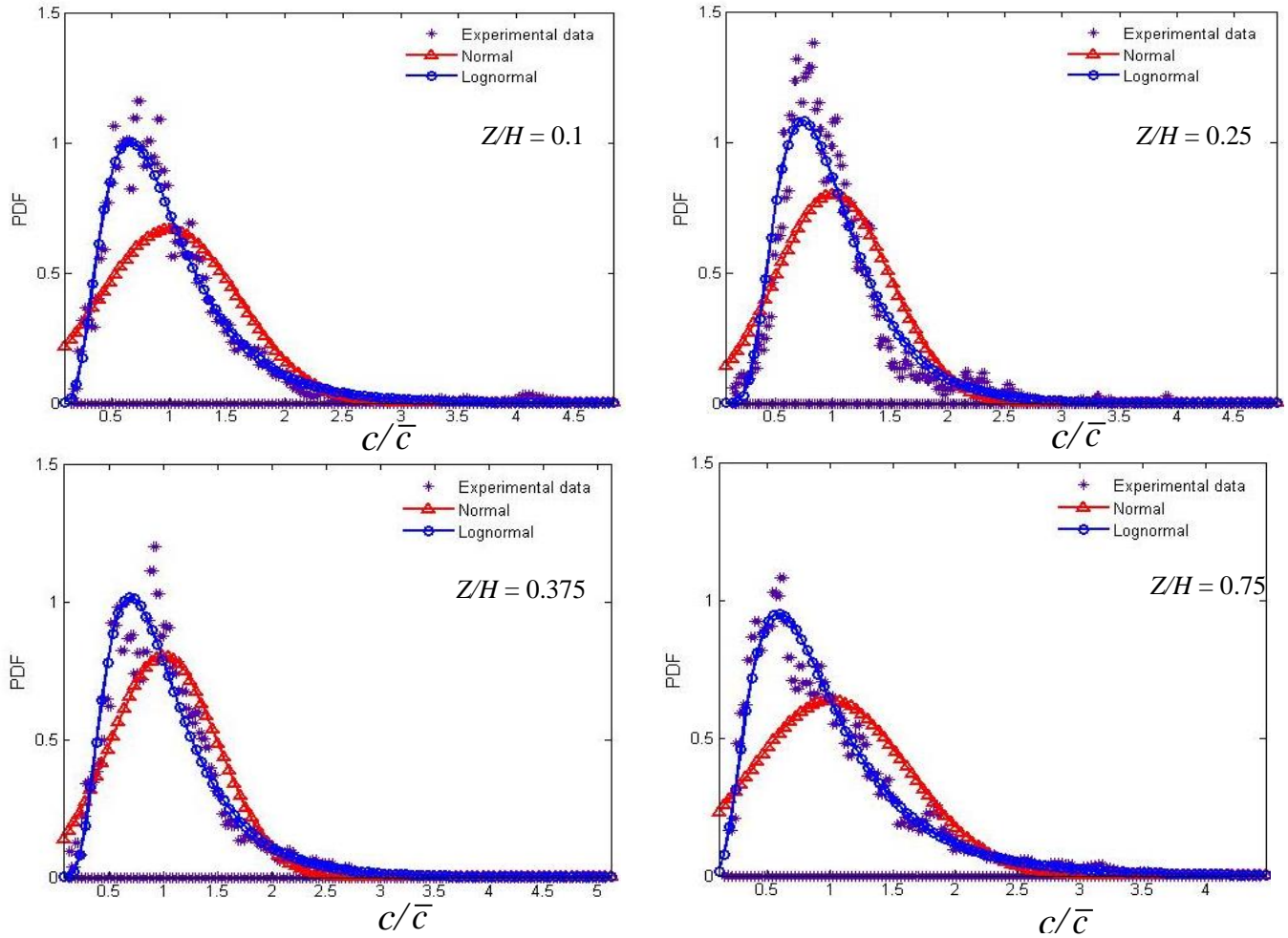

Fig. 5 Probability density for mean concentration measured at $\mathrm{X} / \mathrm{H}=1.125$ 

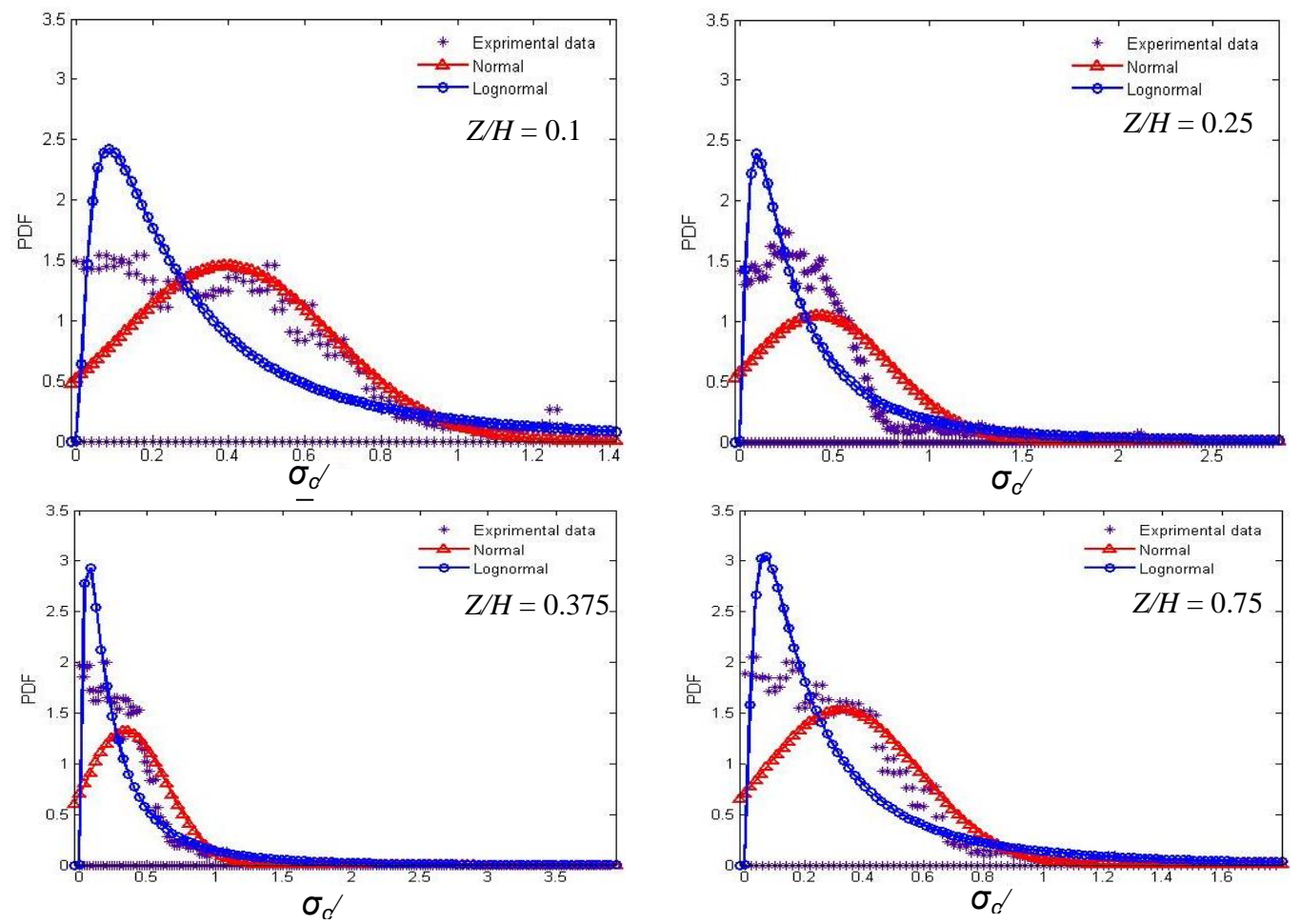

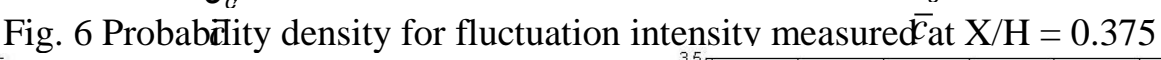
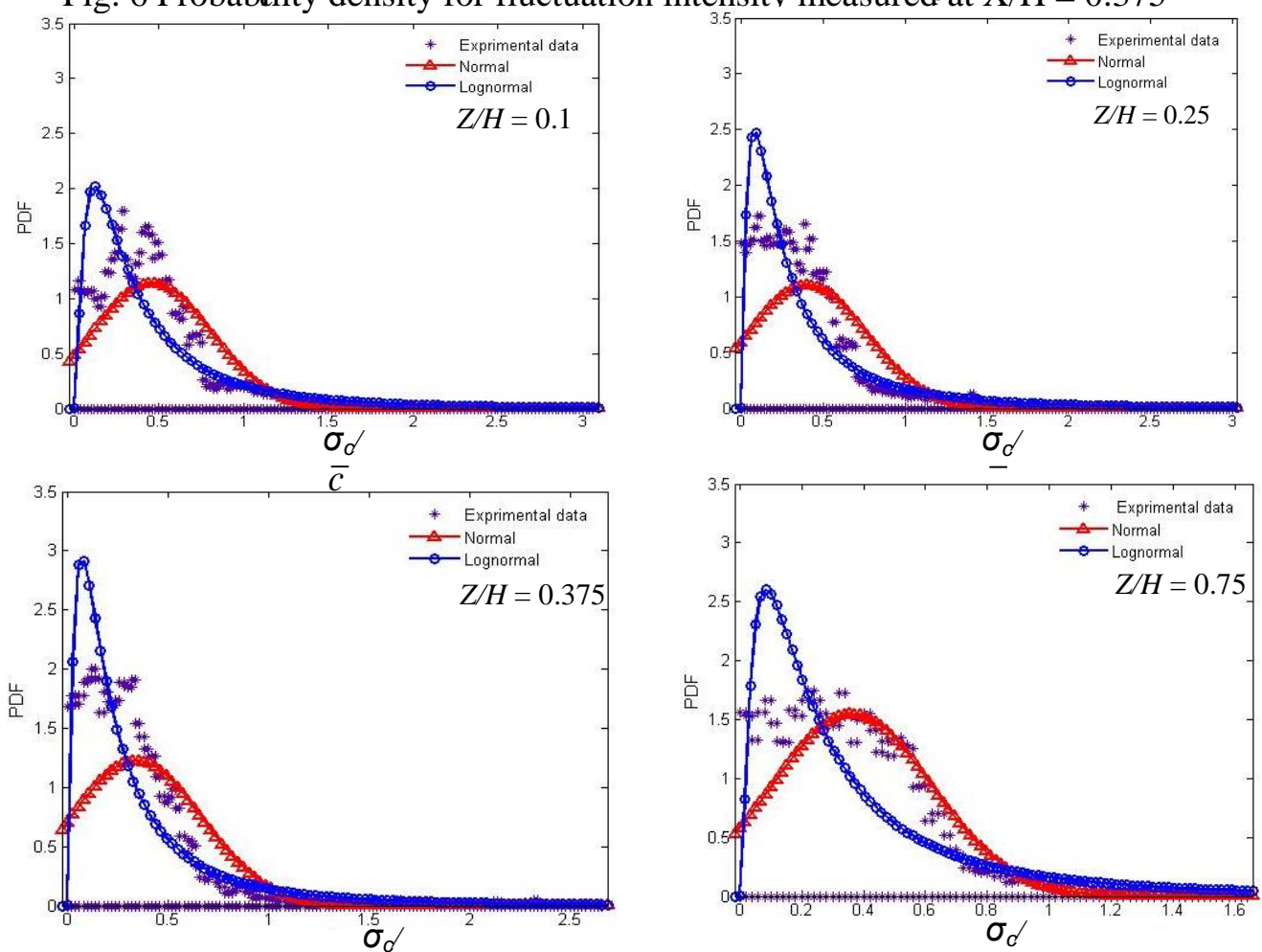

Fig. 7 Probability de $\bar{x}$ sity for fluctuation intensity measured at $\bar{X} / \mathrm{H}=0.625$ 

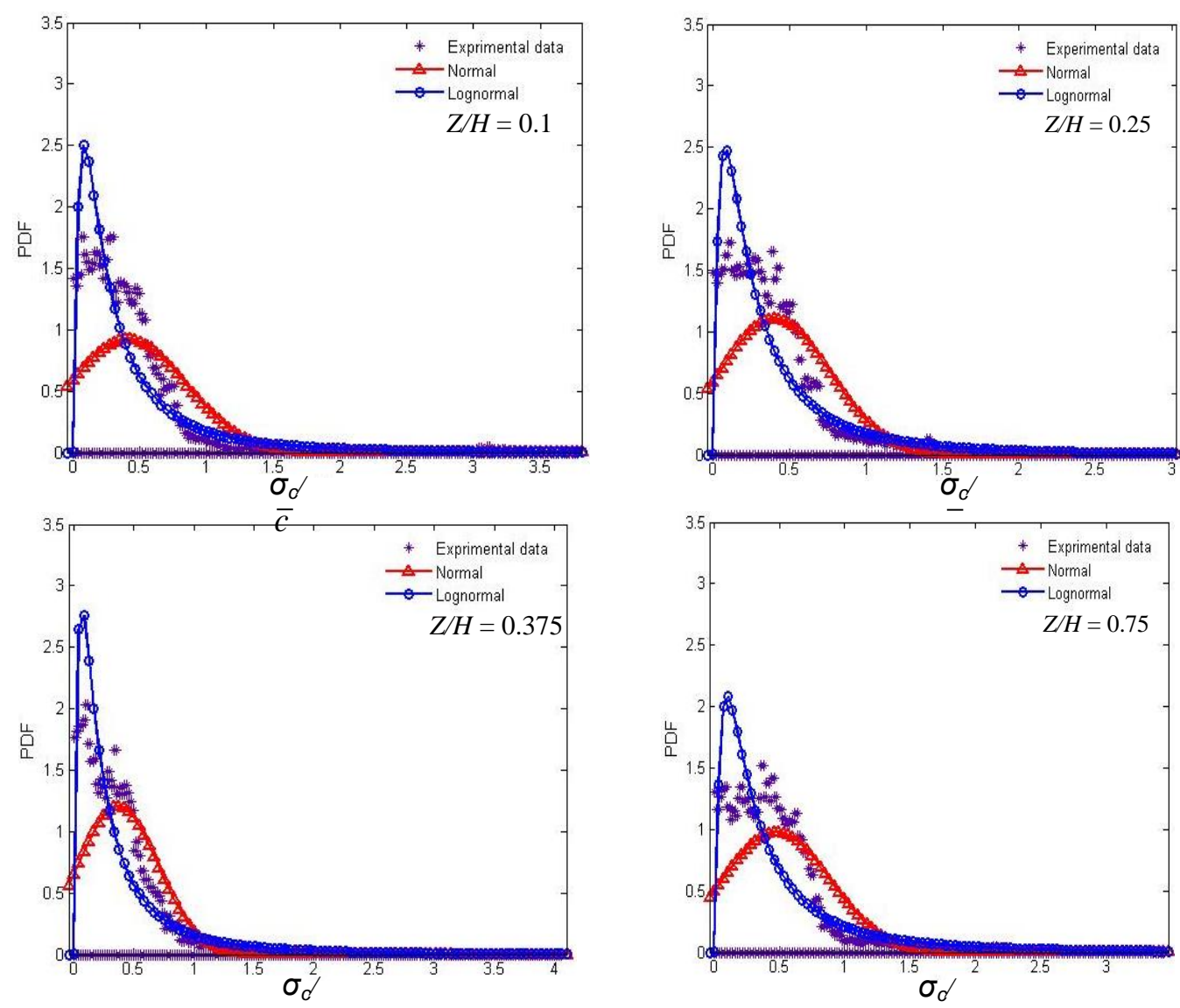

Fig. 8 Probability density for fluctuation intensity measured at $\overline{\mathrm{X}} / \mathrm{H}=1.125$

Table 1: Skewness, Kurtosis and crest factors of fluctuating intensity

\begin{tabular}{|c|c|c|c|c|c|c|c|c|}
\hline $\boldsymbol{X} / \boldsymbol{H}$ & $\boldsymbol{Z} / \boldsymbol{H}$ & \multicolumn{2}{|c|}{ Turbulence intensity, \% } & $\boldsymbol{S}_{\boldsymbol{k}}$ & $\boldsymbol{K}_{\boldsymbol{u}}$ & $\hat{\boldsymbol{c}}$ & $\check{\boldsymbol{c}}$ \\
\cline { 2 - 9 } & & $\boldsymbol{I}_{\boldsymbol{u}}$ & $\boldsymbol{I}_{\boldsymbol{v}}$ & $\boldsymbol{I}_{\boldsymbol{w}}$ & & & & \\
\hline \multirow{3}{*}{0.375} & 0.1 & 16 & 19 & 12 & 0.8081 & 3.4906 & 1.3981 & 0.0001 \\
\cline { 2 - 9 } & 0.25 & 15 & 21 & 16 & 2.3347 & 10.0955 & 2.8158 & 0.0002 \\
\cline { 2 - 9 } & 0.375 & 15 & 17 & 17 & 2.5397 & 16.3599 & 3.8877 & 0.0002 \\
\cline { 2 - 9 } & 0.75 & 14 & 19 & 17 & 1.6799 & 7.3579 & 1.7775 & 0.0003 \\
\hline \multirow{3}{*}{0.625} & 0.1 & 18 & 19 & 14 & 1.9073 & 9.3709 & 3.048 & 0.0003 \\
\cline { 2 - 9 } & 0.25 & 16 & 24 & 18 & 2.2044 & 9.9335 & 2.9957 & 0.0001 \\
\cline { 2 - 9 } & 0.375 & 15 & 20 & 18 & 2.8901 & 14.9268 & 2.6441 & 0.0001 \\
\cline { 2 - 9 } & 0.75 & 15 & 23 & 22 & 1.1867 & 5.1335 & 1.6389 & 0.0001 \\
\hline \multirow{3}{*}{1.125} & 0.1 & 13 & 17 & 16 & 3.7517 & 22.1569 & 3.7791 & 0.0006 \\
\cline { 2 - 9 } & 0.25 & 14 & 20 & 17 & 3.3371 & 20.4092 & 3.8100 & 0.0003 \\
\cline { 2 - 9 } & 0.375 & 14 & 21 & 19 & 2.6463 & 14.9268 & 4.0595 & 0.0001 \\
\cline { 2 - 9 } & 0.75 & 16 & 21 & 21 & 2.2684 & 10.7149 & 3.4216 & 0.0007 \\
\hline
\end{tabular}


there are no currently available data to validate the evolution of the skewness and kurtosis. It can be seen that turbulence intensity in the longitudinal direction $I_{u}$ decrease is associated with an increase in the magnitudes of skewness which is close to the source at $X / H=0.375$, while the turbulence intensity increase with decrease of skewness. Moreover, the shorter tail of fluctuation intensity was appeared with magnitudes of skewness coefficient decrease, while the longer tail was appeared with skewness coefficient increase. The wind tunnel results provide an estimation of the downwind locations of the skewness and they can be useful to drive a suitable parameterization. Close to the source, as the tail is the mean contribution to plume dispersion, the fluctuation Intensity PDF distribution is mainly affected by the largescale motions. The value of kurtosis measured far the source was observed much higher than those obtained close to the source. The value of $\hat{c}$ and č measured far the source at $X / H=1.125$ was observed much higher than those obtained close to the source at $X / H=0.375$ and 0.625 . This illustrates that the concentration fluctuations close to the source shows a higher value of its peak. In comparison of the crest factor $\hat{\mathbf{c}}$ at location close to the source at $X / H=0.375$ and far the source at $X / H=1.125$ with the height at $\mathrm{Z} / \mathrm{H}=0.25$ and 0.375 from $X / H=1.125$. The value of crest factors of fluctuating intensity is low close to the gas source due to the increased longitudinal turbulence intensity $I_{u}$, which create strong meandering plume.

\subsection{Probability Distribution of Crosswind-Plume Dispersion}

Figures 9 and 11 show the probability density functions (PDF) of normalized crosswind plume dispersion $\sigma_{y} / \bar{\sigma}_{y}$ at various distances $X / H=0.375,0.625$ and 1.125 with various heights $\mathrm{Z} / \mathrm{H}=0.1,0.25,0.375$ and 0.75 . From these figures, the distributions obtained for the plume dispersion PDF shows a tendency towards a lognormal distribution of all cases and also positively skewed. The tail of plume dispersion PDF close to the source at distance $X / H=0.375$ for height near the ground surface $Z / H=0.1$ was observed shorter than that those of other tails because the tail of plume is the main contribution to the plume motion. The tail of plume dispersion PDF becomes longer further the source. Moreover, the wider sharp of the plume dispersion PDF was observed at near the source distance $X / H=0.375$ for height $Z / H=0.1$, while the narrow sharp observed gradually further up the source. This phenomenon agrees with previous observations (Hanna and Insley, 1989) that when the plume size is smaller near the gas source, the plume meanders back and forth more due to the advection of atmospheric eddies, resulting in higher intermittency. When the plume is farther away from the gas source and becomes larger than the eddies, they do not meander the whole plume as much but merely cause minor fluctuations deep within the plume, resulting in lower intermittency (Cheung and Melbourne , 2000.). 

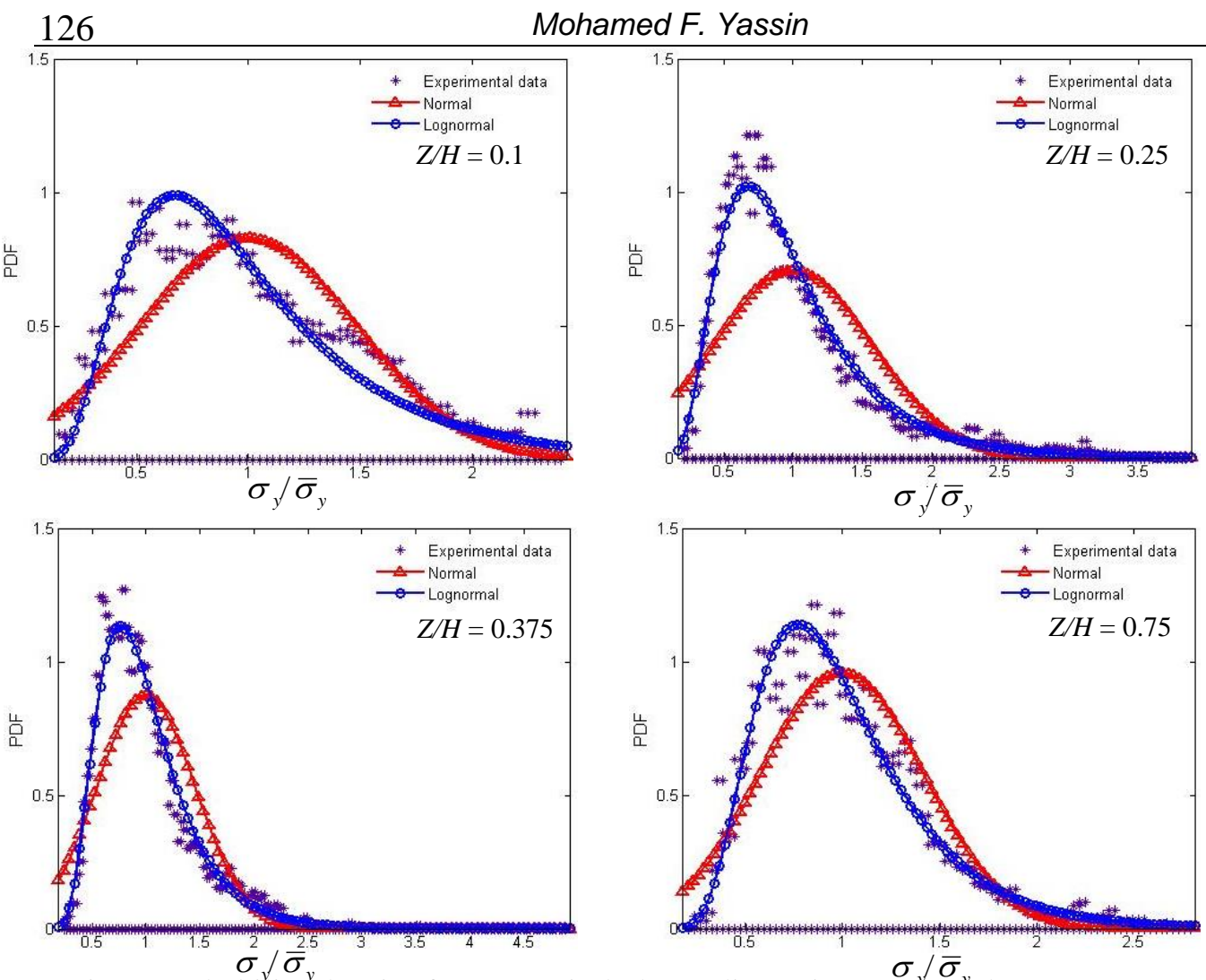

Fig. 9 Probability density for crosswind plume dispersion measured at $\mathrm{X} / \mathrm{H}=0.375$
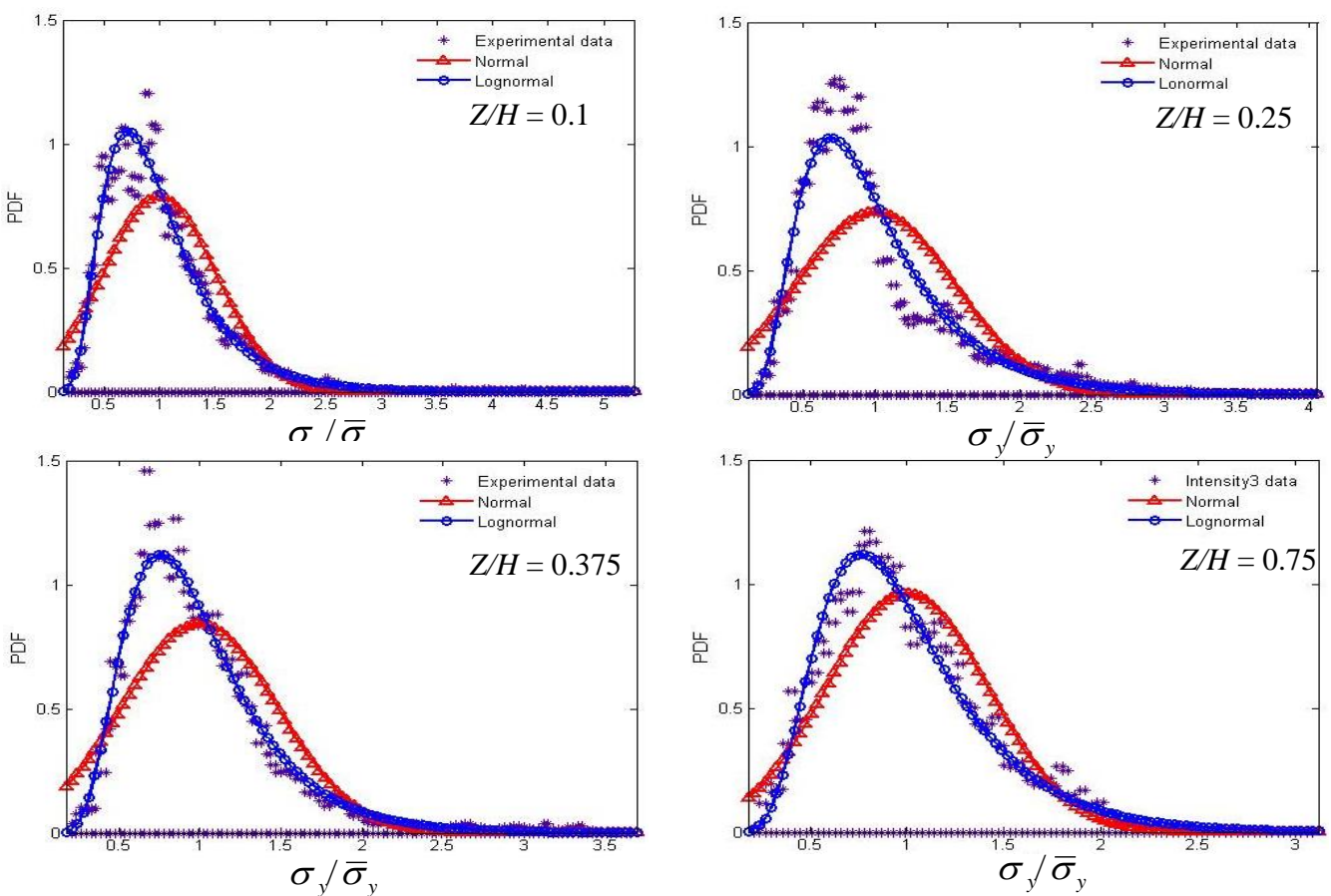

Fig. 10 Probability density for crosswind plume dispersion measured at $\mathrm{X} / \mathrm{H}=0.625$ 

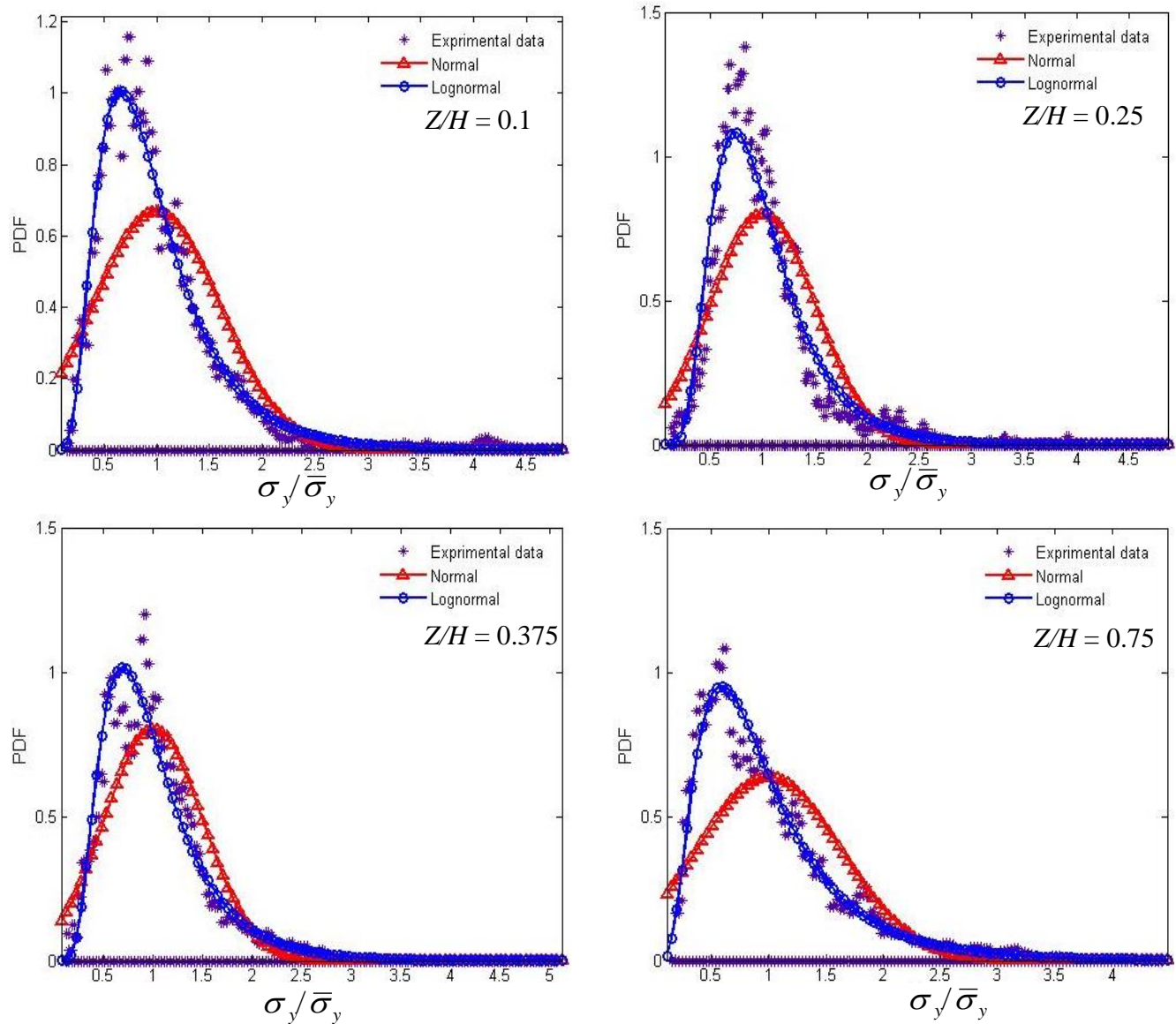

Fig. 11 Probability density for crosswind plume dispersion measured at $\mathrm{X} / \mathrm{H}=1.125$

Table 2: Skewness, Kurtosis and crest factors of crosswind plume dispersion

\begin{tabular}{|c|c|c|c|c|c|c|c|c|}
\hline \multirow[t]{2}{*}{$X / H$} & \multirow[t]{2}{*}{$Z / H$} & \multicolumn{3}{|c|}{ Turbulence intensity, \% } & \multirow[t]{2}{*}{$S_{k}$} & \multirow[t]{2}{*}{$\boldsymbol{K}_{u}$} & \multirow[t]{2}{*}{$\hat{\boldsymbol{c}}$} & \multirow[t]{2}{*}{$\check{c}$} \\
\hline & & $I_{u}$ & $I_{v}$ & $I_{w}$ & & & & \\
\hline \multirow{4}{*}{0.375} & 0.1 & 16 & 19 & 12 & 0.5719 & 2.6323 & 2.3981 & 0.1529 \\
\hline & 0.25 & 15 & 21 & 16 & 1.6278 & 5.9527 & 3.8158 & 0.2333 \\
\hline & 0.375 & 15 & 17 & 17 & 1.5379 & 7.1068 & 4.8877 & 0.2437 \\
\hline & 0.75 & 14 & 19 & 17 & 0.9784 & 4.2088 & 2.7775 & 0.2380 \\
\hline \multirow{4}{*}{0.625} & 0.1 & 18 & 19 & 14 & 1.9539 & 11.3160 & 5.2132 & 0.1884 \\
\hline & 0.25 & 16 & 24 & 18 & 1.5277 & 5.6582 & 3.9957 & 0.1763 \\
\hline & 0.375 & 15 & 20 & 18 & 1.7928 & 7.8732 & 3.6441 & 0.2233 \\
\hline & 0.75 & 15 & 23 & 22 & 0.8579 & 3.9232 & 3.0649 & 0.2193 \\
\hline \multirow{4}{*}{1.125} & 0.1 & 13 & 17 & 16 & 2.3832 & 11.7587 & 4.7791 & 0.1678 \\
\hline & 0.25 & 14 & 20 & 17 & 2.1318 & 10.7010 & 4.8058 & 0.1238 \\
\hline & 0.375 & 14 & 21 & 19 & 1.4626 & 7.6333 & 5.0595 & 0.1238 \\
\hline & 0.75 & 16 & 21 & 21 & 1.4756 & 5.6194 & 4.4215 & 0.1768 \\
\hline
\end{tabular}


Table 2 gives coefficient of skewness and kurtosis which calculated based on the data obtained of crosswind plume dispersion at $X / H=0.375,0.625$ and 1.125 with height $\mathrm{Z} / \mathrm{H}=0.1,0.25,0.375$ and 0.75 . It can be seen that turbulence intensity in the lateral direction $I_{v}$ increase with decrease in skewness for all cases. It is also clear that minimum value of skewness was observed with the shorter tail of the plume dispersion PDF at distance $X / H=0.375$ with $\mathrm{Z} / \mathrm{H}=0.1$. The value of positive crest factor $\hat{\mathrm{c}}$ and kurtosis measured far the source at $X / H=0.625$ and 1.125 were appeared higher than those obtained near the source at $X / H=0.375$.

\section{CONCLUSIONS}

After careful investigation on the probability density distribution of the mean concentration, fluctuation intensity and crosswind mean-plume dispersion in plume dispersion in the neutral boundary layer at various locations and heights in the downwind from the gas source, the results obtained may be summarized as the following: (1) The peak value of concentration PDF was observed at the downwind near the ground in the wake region (2) The lognormal distribution appeared to be the best fit to the shape of the actual probability density functions of fluctuating concentration measured in the boundary layer wind flows in a wind tunnel (3) The tail of concentration PDF for heights far the ground surface was observed shorter than that those heights near the ground surface (4) The fluctuation intensity PDF presented in these figures was clearly positive skewed (5) The fluctuation intensity PDF based on the experimental data are distinctly different from the normal distribution, particularly for both tails (6) The skewness coefficient strongly effects of the tail of fluctuation intensity PDF and (7) The wider sharp of the plume dispersion PDF was observed at distance near the source.

\section{REFERENCES}

1- Alfredo H-S and Wilson H. Tang. Probability concepts in engineering planning and design. John Wiley \& Sons, 1984.

2- Cheung J.C.K., Melbourne W.H. Probability distribution of dispersion from model plume in turbulent wind. Journal of Wind Engineering and Industrial Aerodynamics 2000; 87: 271-285.

3- Diner N., Kaplan H., Kleiman M. Characteristics of concentration fluctuations of a surface plume in neutral boundary layer. Boundary Layer Meteorology 1988; 45: 157-175.

4- Fackrell J.E., Robins A.G. Concentration fluctuation and fluxes in plumes from point sources in a turbulent boundary layer. J. Fluid Mech. 1982; 117: 1-26.

5- Hanna S.R., Insley E.M. Time series analysis of concentration and wind fluctuations. Boundary Layer Meteorology 1989; 47: 31-147.

6- Luhar A.K., Hibberd M.F., Borgas M.S. Askewed meandering plume model for concentration statistics in the convective boundary. Atmospheric Environment 2000; 34: 3599-3616.

7- Li Q.S., Calderone, Melbourne W.H. Probabilistic characteristics of pressure fluctuations in separated and reattaching flows flow various free-stream 
turbulence. Journal of Wind Engineering and Industrial Aerodynamics 1999; 82: 125-145.

8- Ma Y., Boybeyi Z., Hanna S., Chayantrakom K. Plume dispersion from the MVP field experiment: Analysis of surface concentration and its fluctuation. Atmospheric Environment 2005; 39: 3029-3054.

9- Mfula, A.M., Kukadia, V., Griffiths, R.F. and Hall, D.J. Wind tunnel modeling of urban building exposure to outdoor pollution. Atmospheric Environment 2005; 39: 2737-2745.

10- Mylne K.R. Concentration fluctuation measurement in a plume dispersing in a stable surface layer. Boundary Layer Meteorology 1992; 60: 15-48.

11- Mylne K.R., Mason P.J. Concentration fluctuation measurements in dispersion at a range of up to 1000m. Q.J.R. Meteorol. Sco. 1991; 117: 177-206.

12- Sawford B.L., Frost C.C., Allan T.C. Atmospheric Boundary layer measurements of concentration statistics from isolated and multiple sources. Boundary Layer Meteorology 1985; 31: 249-268.

13- Sykes R.I. The variance in time average samples from an intermittent plume. Atmospheric Environment 1984; 18: 121-123.

14- Sykes R.I. Concentration fluctuations in dispersion plumes. In: Venkatram A. ,Wyngaard J.C., Boston, editors. Lecture on Air Pollution Modeling, 1988, pp. 325-356.

15- Hanna S.R., 1984. Concentration fluctuations in smoke plume. Atmospheric Environment 1984; 18, 6: 1091-1984.

16- Weil J.C., Snyder W.H., Lawson Jr R.E., Shpman M.S. Experiments on buoyant plume dispersion in a laboratory convection tank. Boundary Layer Meteorology 2002; 102: 367-414.

17- Yassin M.F., Ohba M., Tanaka H. (2006) 'The influence of obstacles on the flow and pollutant dispersion in an Urban Environment' $14^{\text {th }}$ joint conference on application of air pollution meteorology with the air and waste management Assoc, Atlanta, Georgia, USA, Jan. 29 -Feb. 2.

18- Yee E., Biltoft C.A. Concentration fluctuation measurements in a plume dispersing through a regular array of obstacle. Boundary Layer Meteorology 2004; 111: 363-415.

19- Yee E., Chen R. A simple model for the probability density function of concentration fluctuations in atmospheric plume. Atmospheric Environment 1997; 31: 99-1002. 


\section{الخصائص المحتملة للتركيزٍ المتنّبذب في تثتت الريثةِ}

الخصائص المحتملـة للتركيز المتذبـذبِ في نتشت الريشـةِة بحثت عمليا في نفق هوائي معملي خلال

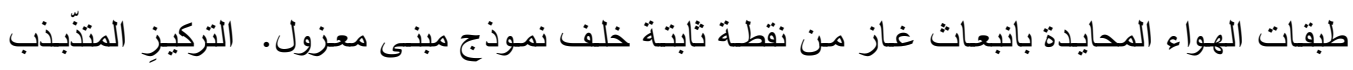

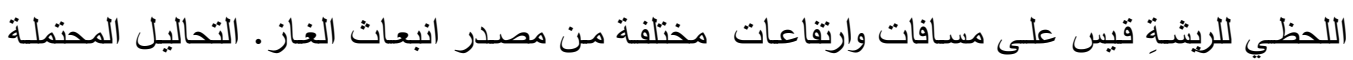

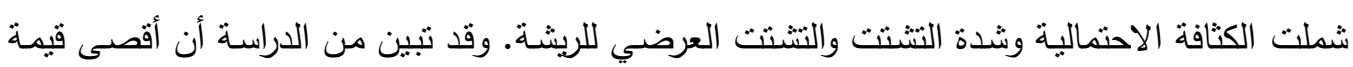

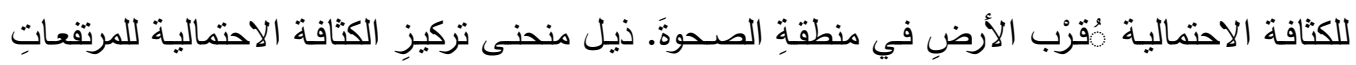

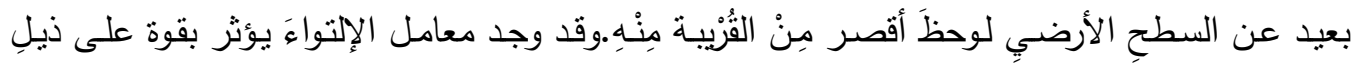

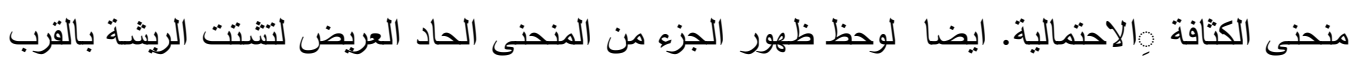

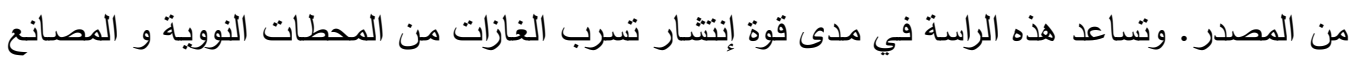
كيماوية. 